\title{
Erratum: On the Dirichlet problem for minimal graphs in hyperbolic space
}

\section{Fanghua Lin}

Published online: 29 November 2011

(C) Springer-Verlag 2011

\section{Erratum to: Invent Math (1989) 96: 593-612 DOI 10.1007/BF01393698}

Let $H^{n+1}=\left\{(x, y) \in \mathbb{R}^{n} \times R, y>0\right\}$ denote the hyperbolic space equipped with the metric

$$
d s^{2}=\frac{d x^{2}+d y^{2}}{y^{2}} .
$$

In this model, $H_{\infty}^{n+1}=\mathbb{R}^{n} \times\{0\} \cup\{*\}$ is the one-point compactification of $\mathbb{R}^{n} \times\{0\}$ that can be identified with the asymptotic infinity of $H^{n+1}$.

Let $N^{n-1} \subset H_{\infty}^{n+1}$ be a closed embedded $(n-1)$-dimensional submanifold. Then there exists a complete absolutely area-minimizing integral $n$-current $\Sigma$ asymptotic to $N^{n-1}$ at infinity; see, for example [1, 2]. It was also noted in $[1,2]$ that in the case $n \leq 6$, currents obtained are smoothly embedded complete hypersurfaces. In the case $n \geq 7$, as in the Euclidean case, there can be a singular set of Hausdorff dimension at most $n-7$.

This erratum should have been written nearly twenty years ago [5]. I apologize for any inconvenience or confusion that may have resulted from the lengthy delay in its composition.

The online version of the original article can be found under doi:10.1007/BF01393698.

F. Lin (凶)

Courant Institute of Mathematical Sciences, New York University, 251 Mercer Street, New York, NY 10012-1185, USA

e-mail: linf@cims.nyu.edu 
The question of the boundary behavior of such hypersurfaces was discussed in [3]; see also [4] for the case of general higher codimension. It was shown that if $N$ is a $C^{1}$-submanifold of $H_{\infty}^{n+1}$, then $(\Sigma \cup N) \cap\left\{y<\rho_{N}\right\}$ is a finite union of $C^{1}$-submanifolds with boundary for some $\rho_{N} \geq 0$. Moreover, these $C^{1}$-submanifolds have disjoint analytic interiors and meet $\mathbb{R}^{n} \times\{0\}$ orthogonally at $N$.

Concerning higher-order regularity, the following result was claimed in [5].

Theorem [5] Suppose that $N^{n-1}$ is $C^{k, \alpha}$ with either $1 \leq k \leq n-1$ and $0 \leq \alpha \leq 1$ or $k \geq n$ and $0<\alpha<1$. Then there is a positive $\rho_{N}$ such that $(\Sigma \cup N) \cap\left\{y<\rho_{N}\right\}$ is a $C^{k, \alpha}$ submanifolds hypersurface with boundary.

This result is not fully correct. The proofs given in [5] for the case when either $1 \leq k \leq n-1$ and $0 \leq \alpha \leq 1$ or $k=n$ and $0<\alpha<1$ were correct. But the proof in [5] for the case $k \geq n+1$ was incorrect; in fact, the result is not true for $k \geq n+1$ when $n \geq 3$ is odd. The correct statements as well as the proofs for the case $k \geq n+1$ were provided in Y. Tonegawa's 1993 Ph.D. thesis at New York University [6], and part of that thesis related to these results appeared in [7].

For the convenience of readers, we summarize the full and correct statements as follows:

Theorem Suppose that $N^{n-1}$ is of class $C^{k, \alpha}$.

(I) If $1 \leq k \leq n-1$ and $0 \leq \alpha \leq 1$ or $k=n$ and $0 \leq \alpha<1$, then $M=$ $(\Sigma \cup N) \cap\left\{y<\rho_{N}\right\}$ is a $C^{k, \alpha}$ hypersurface with boundary.

(II) If $k \geq n+1$ and $0<\alpha<1$, then,

(IIa) for $n$ even, $M$ is a $C^{k, \alpha}$ hypersurface with boundary;

(IIb) for $n$ odd, there is a necessary and sufficient condition on $N$ (a differential equation of order $(n+1)$ to be satisfied by $N)$ so that $M$ will be a $C^{k, \alpha}$ hypersurface with boundary provided this additional condition is satisfied.

\section{References}

1. Anderson, M.T.: Complete minimal varieties in hyperbolic space. Invent. Math. 69(3), 477494 (1982)

2. Anderson, M.T.: Complete minimal hypersurfaces in hyperbolic $n$-manifolds. Comment. Math. Helv. 58(2), 264-290 (1983)

3. Hardt, R., Lin, F.-H.: Regularity at infinity for area-minimizing hypersurfaces in hyperbolic space. Invent. Math. 88(1), 217-224 (1987)

4. Lin, F.-H.: Asymptotic behavior of area-minimizing currents in hyperbolic space. Commun. Pure Appl. Math. 42(3), 229-242 (1989)

5. Lin, F.-H.: On the Dirichlet problem for minimal graphs in hyperbolic space. Invent. Math. 96(3), 593-612 (1989) 
6. Tonegawa, Y.: Complete constant mean curvature surfaces in hyperbolic space. Ph.D. thesis, New York University, 99 pp., ProQuest LLC, Thesis. Available at: http://proquest.umi.com/pqdlink?Ver=1\&Exp=07-27-2016\&FMT=7\&DID=745772511\& RQT $=309 \&$ attempt=1\&cfc=1 (1993)

7. Tonegawa, Y.: Existence and regularity of constant mean curvature hypersurfaces in hyperbolic space. Math. Z. 221(4), 591-615 (1996) 